\title{
The effect of shift-working conditions of nurses on health
}

\author{
Canan Birimoglu Okuyan ${ }^{1 *}$ and Ebru Deveci ${ }^{2}$ \\ ${ }^{1}$ Mustafa Kemal University, Hatay Health School, Tayfur Sökmen Campus, 31060 Alahan-Antakya/Hatay, Turkey \\ ${ }^{2}$ Hasan Kalyoncu University, Vocational School of Health Services, Turkey
}

\begin{abstract}
The working in a shift system is a potential risk factor for the occurrence of psychiatric disorders and low quality of life in nurses. Therefore, regulation of working hours by taking into consideration the problems that may occur in employees will contribute to improving the quality of life. This paper focuses on the effect of shift-working conditions of nurses on health.
\end{abstract}

Working life is an indispensable part of human life. Working environments in which the most active period of the day is experienced can affect physical, mental, and social health due to the insatiability, accidents and risks that may disrupt the employee's health. For this reason, the characteristics of the working environment are very important in protecting and maintaining the health of employees [1].

Working environment influences nurses as in all professions. Nursing is not only a stressful occupation with an intense workload but also has many negative factors arising from the working environment. The nurse is the first person to whom the patient and his/her family apply for all kinds of problems within 24 hours and the health personnel who play a key role by providing communication within the health team because of this duty. It is of utmost importance that the nurses who provide health services to people should be in a kind of wellbeing and that this kind of wellbeing is protected. Having a dynamic structure of the nursing profession requires continuous work and continuous service, that is to say working in shifts. This situation negatively affects the attention and productivity in nurses who have to work in a shift system because the hospitals are provided with uninterrupted health services. The problems experienced in the working environment affect the nurses negatively in physical, psychological, and social aspects. Errors to be made can lead to results that cannot be compensated [2]. The most important of these problems is the problem of sleep. It was found that there was a significant relationship between sleepiness and work accidents [3]. Working time at the workplace and the frequency and duration of rest periods are highly influential on the physical and mental balance of employees [4]. Sönmez et al. found that there was a significant relationship between sleepiness and work accidents in nurses working in shifts [3].

Working in shifts affects social life in addition to physiological and mental conditions. It negatively affects the irregular working arrangements, especially marital and child-related responsibilities of women working in shifts, due to insufficient time constraints. In workers working in shifts, disruption of family order, divisions, weakness in friendship, and a number of problems arise [5]. Ruggiero and Janino pointed out working on weekends and special days negatively affects the social lives of individuals [6]. When an employee is particularly a woman, working in shifts can cause more specific and negative effects on women's health, such as hormonal activity, and production system functions. Working in shifts not only affect nurses' health problems and their quality of life but also decrease the efficiency of work and cause the economic loss in the institutions that they are working for due to absenteeism to and put the individuals they care for into risk. This can go on until the nurses quit their jobs. Schönfelder and Knauth have pointed out, working in shifts leads to negative effects on the health, well-being, social life, physiological adjustment and job performance of the nurse in terms of the results [7]. In a study by Kesgin and Kublay, it was found that working in a shift system negatively affects the development of positive health habits of nurses [8].

In the study of Çalık et al. conducted with 477 nurses in Trabzon, $87.4 \%$ of the nurses reported that the working hours have one or more negative effects on social and family life, $87.2 \%$ of psychological health, $95.2 \%$ on physical health and $77.6 \%$ on their own safety [9]. It is useful and necessary to make some practices to protect and improve the health of the nurses who spend a large part of the day in the hospital and the patients they serve in the health system in which it is inevitable to work in the shift system. Firstly, the shift work schedule should be arranged in such a way that it does not adversely affect the quality of work and productivity. For the hospitals with 24 hours of continuous service, the most suitable shift system is the 8 hour triple shift system in daytime-evening-night mode. In addition, it should be ensured that the next shifting hour has to be arranged as having at least 24 hours for resting, and the weekly working time must not exceed 40 hours. Working conditions should be created to provide nurses satisfaction with working life quality, and should be arranged in accordance with the human needs of work processes, working conditions and environment. Additional nurses other than the existing nurses should be employed to meet the over-service need arising from increased patient density in the service. In addition, adverse conditions arising from shift working conditions should be regulated by laws and codes. For the solution of problems related to social life and home life of nurses resulting from working in shift; The shift start and end time, the shift time and the speed of shift conversion should have a specific standard in accordance with ergonomic norms, which will not differ from the hospital to the

Correspondence to: Canan Birimoğlu Okuyan, Mustafa Kemal University, Hatay Health School, Hatay, Turkey, Tel: +905539272035; E-mail: cananbirimoglu@gmail. com

Key words: nurse, shift, working conditions

Received: September 20, 2017; Accepted: October 09, 2017; Published: October 11,2017 
hospital. The fee paid for the night in the shift system should be above the fee paid for daytime work.

In addition to institutional and legal regulations, the meaning that nurses and their families put into the work and working in shift is also very important. With the support of the family and social environment, nurses may feel less negative impacts of working in shifts on the social life and home life. Hearty friend relationships can reduce the pressure of working in shifts. In addition, nurses' being busy with things they love outside of working hours and getting involved in sportive and cultural activities may also reduce the negative effects of working in shifts. The protection of mental health depends primarily on the elimination of physiological needs and disorders. Nurses should also protect their health status by having regular health checks. After the intense and sleepless shift, they should take time to rest, feed regularly and healthily.

\section{References}

1. Dindar İ, İşsever H, Özen M (2004) Discomforts and Problems Related to the Isle of Nurses in the Hospitals of Edirne. Journal of Nursing Forum Magazine 7.
2. Sezgin B (2007) Evaluation of work environment and patient and nurse safety of nursing practices in hospitals which have received quality certificate Ph.D. Thesis, İstanbul University, Health Science Institutes, İstanbul.

3. Sönmez S, Ursavaş A, Uzaslan E, Ediger D, Karadağ M, et al. (2010) Sleep Disorders and Occupational Accident in Shift Work Nurses. Journal of Turkish Toraks 11: 105-108.

4. Camkurt MZ (2007) The Impact of Workplace Work System and Workplace Physical Factors on Job Accidents. TÜHIS-Journal of Business Law and Economics 20.

5. Arpacı FA (2007) Study into the Effects of Shift Work on Social and Domestic Lives of Nurses. New World Sciences Academy 2.

6. Ruggiero J, Pezzino (2006) Nurses Perceptions of the Advantages and Disadvantages of Their Shift and Ork Shedules. JONA 36: 450-453. [Crossref]

7. Schönfelder E, Knauth PA (1993) Procedure to Assess Shift Systems Based on Ergonomic Criteria. Ergonomics 36: 65-76.

8. Kesgin MT, Kubilay G (2011) The Evaluation of Healthy Problems Caused from Working Conditions and Life Habits of Nurses that Working in a Private Hospital. Journal of Hacettepe University Faculty of Nursing. 18: 041-049.

9. Çalık KY, Aktaş S, Bulut HK, Anahar EÖ (2015) The Effects of Shift Working and Rotating Shifts on Nurses. Journal of Health Science and Profession 2: 33-45.

Copyright: $\left(\mathbb{C}_{2017}\right.$ Okuyan CB. This is an open-access article distributed under the terms of the Creative Commons Attribution License, which permits unrestricted use, distribution, and reproduction in any medium, provided the original author and source are credited. 